\title{
Hydraulic Performance of an S-Draft Tube for a Low-Head Axial Flow Turbine* (How to Improve the Performance and Unsteady Secondary Flow in a S-Draft Tube)
}

\author{
Yukimaru SHIMIZU**, Takashi KUBOTA***, \\ Yoshisato WAKASHIMA** and Shogo NAKAMURA***
}

\begin{abstract}
This study investigates the possibility of significantly improving the performance of sharply bent S-draft tubes by a conventional, but comparatively simple method ; by adjusting the divergent angle of the conical straight portion at the upstream part of the draft tube. This paper describes this method, as well as a phenomenon associated with secondary flow in sharply bent S-draft tube in which the flow changes its direction intermittently. This phenomenon is important because the use of sharply bent $\mathrm{S}$-draft tubes is preferred in some cases due to civil engineering requirements for turbine installation. Therefore, this report attempts to clarify the phenomenon in detail.
\end{abstract}

Key Words: Hydraulic Machine, S-Shaped Draft Tube, Low-Head Water Turbine, Improvement of Pressure Recovery Coefficient, Unsteady Secondary Flow, Sharp Bend

\section{Introduction}

At the previous 12 th IAHR $^{(1)}$, the authors presented a study clarifying the relationship between pressure recovery performance and geometrical shape of S-draft tubes, and the relationship between velocity distribution and pressure recovery performance of $\mathrm{S}$ tubes, which are used for low-head axial flow turbines. The study explained the pressure recovery performance of four types of S-draft tubes.

Subsequent study has revealed a phenomenon that the secondary flow in sharply bent S-draft tube changes its rotational direction intermittently. This phenomenon is important because the use of sharply bent $\mathrm{S}$-draft tubes is preferred in some cases due to civil engineering requirements for turbine installation. Therefore, this report attempts to clarify the phenomenon in detail.

* Received 18th January, 1988. Paper No.86-0901A

* * Department of Mechanical Engineering, Mie University, Kamihama-cho, Tsu, Mie, 514, Japan

* * Hydro-Turbine Division, Fuji Electric Co., Ltd., 1-1 Tanabeshinden, Kawasaki-ku, Kawasaki, 210, Japan
We have further confirmed the possibility of improving the performance of sharply bent S-draft tubes significantly by a conventional, but comparatively simple method; by adjusting the divergent angle of the conical straight portion at the upstream part of the draft tube. A description of this method is also given in this paper.

\section{Nomenclature}

$A R$ : Area ratio for annular inlet section, = $A_{n} / \pi\left(r_{w 1}{ }^{2}-r_{b}{ }^{2}\right)$

$A_{n}:$ Area of $n$-th section

$\tilde{C}_{p}$ : Dimensionless value of wall pressure distribution; [Eq. (3)]

$C_{p o}$ : One-dimensional pressure recovery coefficient ; [Eq. (1)]

$M_{1}^{\prime}$ : Swirl flow intensity at inlet; [Eq. (2)]

$P_{w 1}:$ Wall pressure at inlet

$P_{w n}:$ Pressure at $\mathrm{n}$-th wall pressure hole

$\bar{P}_{r}$ : Average pressure at outlet section

$Q:$ Flow rate measured with orifice meter

$r$ and $r_{w 1}$ : Radial position and radius to the wall at inlet

$r_{b}$ : Radius of boss at inlet portion $(=11.6 \mathrm{~mm})$ 


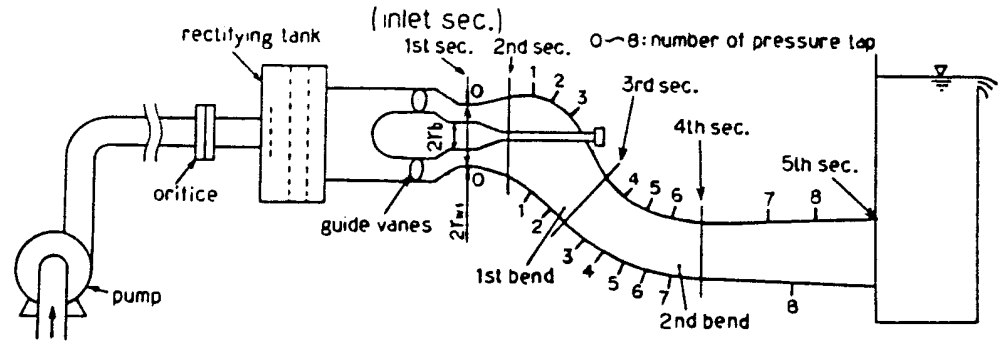

Fig. 1 Schematic diagram of experimental apparatus

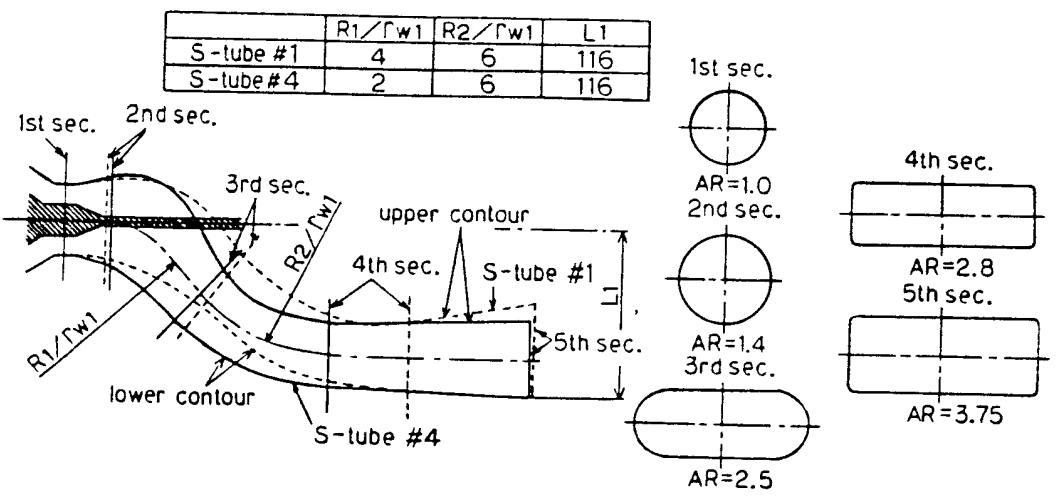

Fig. 2 Model S-tubes \#1 and $\# 4$

$V_{z n}$ and $V_{a n}$ : Axial velocity and peripheral velocity at $n$-th section

$V_{m 1}:$ Average axial velocity at inlet section

$\alpha:$ Angular position within the section ( $\mathrm{rad}$ )

$\rho$ : Density of water

\section{Experimental Apparatus and Method}

The experimental apparatus is outlined in Fig. 1. Water is pumped into the rectifying tank via an orifice meter which measures the flow rate. The water flows into the draft tube through the runway and vortex generator containing six guide vanes. Figure 2 shows the S-draft tubes used for the experiment: the thin broken line represents the \# $1 \mathrm{~S}$-draft tube, while the thick solid line indicates the sharply bent \#4 S-draft tube. The latter type of S-draft tube is sometimes required when space limitations are encountered at turbine locations. In the experiment, the divergent angle of the conical straight diffuser (see Fig. 3) at the inlet of the draft tube was varied from 12 degrees to 24 degrees or 36 degrees, and the effects of these angles on pressure recovery performance were studied.

Wall pressure distribution was measured at points 0 through 8 on the upper and lower tube walls as shown in Fig. 1. For determining the No. 0 value, four holes were provided on the circumference, and

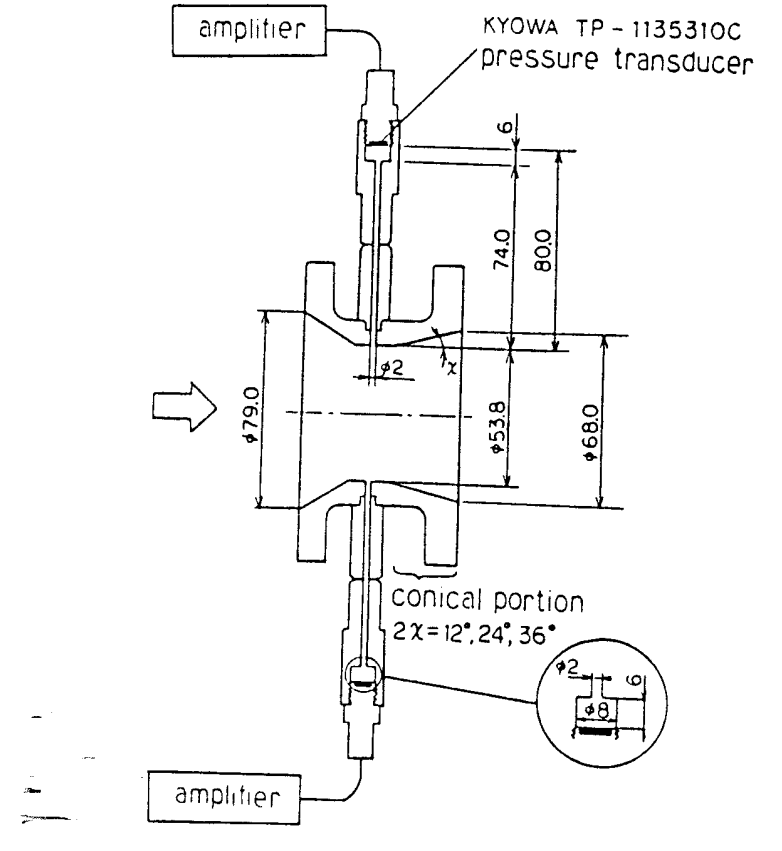

Fig. 3 Explanation of measurement of pulsating pressure and feature of inlet conical diffuser. Pressure transducers are used.

the four point values were averaged. Then, pressures at points 1 through 8 were measured against that of point 1 using a manometer. In order to clarify the 
influences of value differences among the No.0 circumferential points, especially between the upper and the lower points, the differences between the upper hole pressure and the lower hole pressure and between the average No. 0 pressure value and the wall pressures were compared, but the difference was negligible. To measure pulsating pressure, the pressure transducers were set on the No.0 pressure hole shown in Fig. 3. Hole diameter was $2 \mathrm{~mm}$, which was sufficient to transfer pulsating pressure. Tuft-grid and surface-tuft methods were used to visualize the flow in the S-draft tube. In the case of flow visualization, air flow was used. The experiment was conducted at a Reynolds number of approximately $1.8 \times 10^{5}$.

\section{Definition of Parameters}

When the performance of a draft tube is discussed, the one-dimensional pressure recovery coefficient $C_{p o}$ is often used, which is expressed by the following equation:

$$
C_{p o}=\left(\bar{P}_{T}-P_{w 1}\right) / \frac{\rho}{2} V_{m i}^{2}
$$

The intensity of swirling flow at the inlet section is expressed by the dimensionless angular momentum flow rate as follows:

$$
M_{1}^{\prime}=\frac{\int_{0}^{2 \pi} \int_{r_{b}}^{r_{w 1}} v_{z 1} v_{a 1} r^{2} d r d a}{r_{w 1} \pi\left(r_{w 1}{ }^{2}-r_{b}^{2}\right) V_{m 1}^{2}}
$$

Dimensionless wall pressure difference is defined as follows:

$$
\tilde{C}_{p}=\left(P_{w n}-P_{w 1}\right) / \frac{\rho}{2} V_{m !}^{2}
$$

\section{Experimental Results}

\subsection{Pressure recovery performance of sharply} bent $\mathrm{S}$-draft tube

In some installations of a low-head axial flow turbine with an S-draft tube, the space conditions require that the tube be bent at a sharp angle. Figure 4 shows the pressure recovery coefficient of the $\$ 1 \mathrm{~S}$ draft tube with good performance and the pressure re-

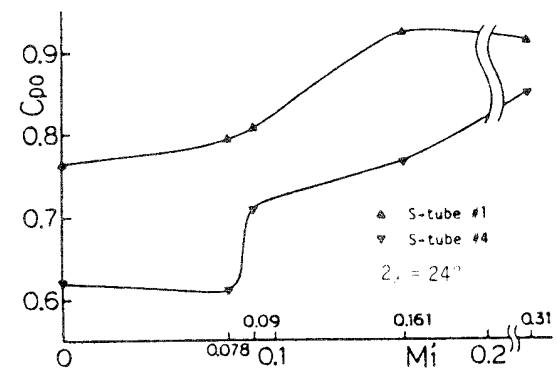

Fig. 4 Relation between one-dimensional pressure recovery performance $C_{\rho_{0}}$ and swirl intensity of inlet flow $M_{i}^{\prime}$ at the inlet section covery coefficient of the sharply bent $\# 4 \mathrm{~S}$-draft tube.

Phenomena related to the pressure recovery coefficient of the sharply bent $\# 4 \mathrm{~S}$-draft tube was investigated by varying swirl flow intensity $\left(M_{1}^{\prime}\right)$ at inlet of the draft tube. In case of a sharply bent draft tube, pressure recovery coefficient is low $C_{p o} \fallingdotseq 0.62$ ) when the swirl flow intensity $\left(M_{1}^{\prime}\right)$ is below $0.078 . C_{p o}$ is improved sharply to 0.71 when $M_{1}^{\prime}$ increases to 0.09 . Thereafter, $C_{p o}$ increases gradually as $M_{1}^{\prime}$ increases. In the case of a gently curved S-draft tube, $C_{p o}$ is 0.77 at $M_{1}^{\prime}=0,0.79$ at $M_{1}^{\prime}=0.078$, and thereafter increases gradually as $M_{i}^{\prime}$ Increases. Such differences between the two draft tubes in pressure recovery coefficient depends on the area of separation zone formed on the inner side of the curvature at the first bend shown in Fig. 1.

Figure 5 presents visualized photographs of the flow in the third section of the sharply bent $\# 4$ S-draft tube using the Tufts-Grid method. In Pic. 1, showing the case of $M_{1}^{\prime}=0$, large separation is seen on the inner side of the curvature, and the outer side of the curvature, which shows no separation, is governed by the secondary flow, the right and left halves of which are symmetrical. Picture 2 is a visualized photograph showing a medium uni-directional swirl flow at $M_{1}^{\prime}=$ 0.16 . When a uni-directional swirl flow with a certain intensity exists, as in this case, the separation at the inner side of curvature is suppressed, and most of the
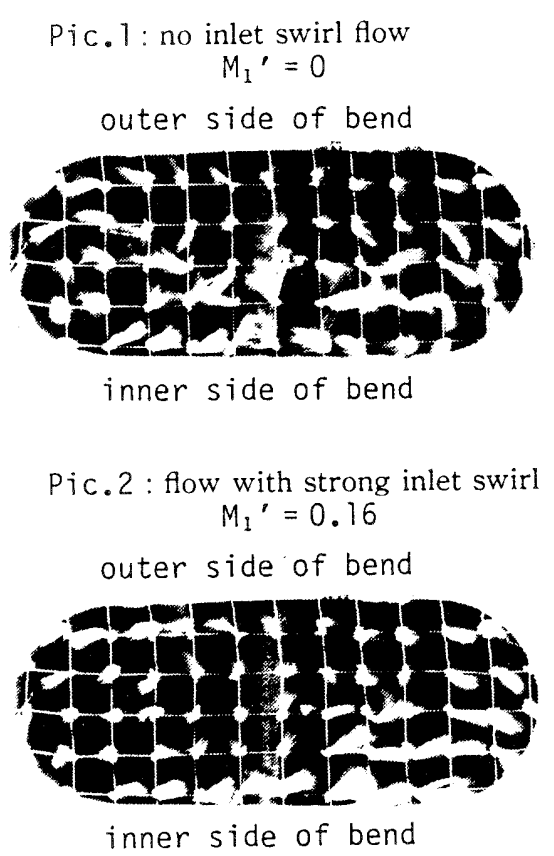

Fig. 5 Pictures of flow visualization in the 3 rd section (the outlet of the 1 st bend) of S-tube $\# 4$ by means of tufts. 
section is governed by the large uni-directional swirl flow. The small reverse swirl flow, which is observed at a right corner in the section, is not influential. As the separation is suppressed as mentioned above or the separation area decreases significantly, the pressure recovery performance is improved.

\subsection{Method for preventing performance decrease of sharply bent draft tubes}

It was revealed that in case of small inlet swirling the pressure recovery performance decreases significantly if the first bend of the S-draft tube is sharply bent. The authors experimentally examined whether it was possible to avoid the decrease of performance. As a result, we succeeded in improving the performance by a comparatively simple method which is described below.

For the draft tube shown in Fig. 2, the relationship between the divergent angle of the conical straight portion at the inlet and the pressure recovery performance was examined. The experiment was conducted at divergent angles of $2 \chi=12^{\circ}, 24^{\circ}$ and $36^{\circ}$. Figures $6(\mathrm{a})$ and ( $\mathrm{b}$ ) show the results: (a) indicates an S-tube with a gentle curvature, and (b) shows $\mathrm{S}$-tube with sharp curvature. Both (a) and (b) indicate that $C_{p o}$ is maximum at $2 \chi=12^{\circ}$, and $C_{p o}$ becomes smaller as the divergent angle increases. In the case of \#1 S-draft tube having good performance with $2 \chi=12^{\circ}, C_{p o}$ is 0.84 at $M_{1}^{\prime}=0$, and $C_{p o}$ reaches
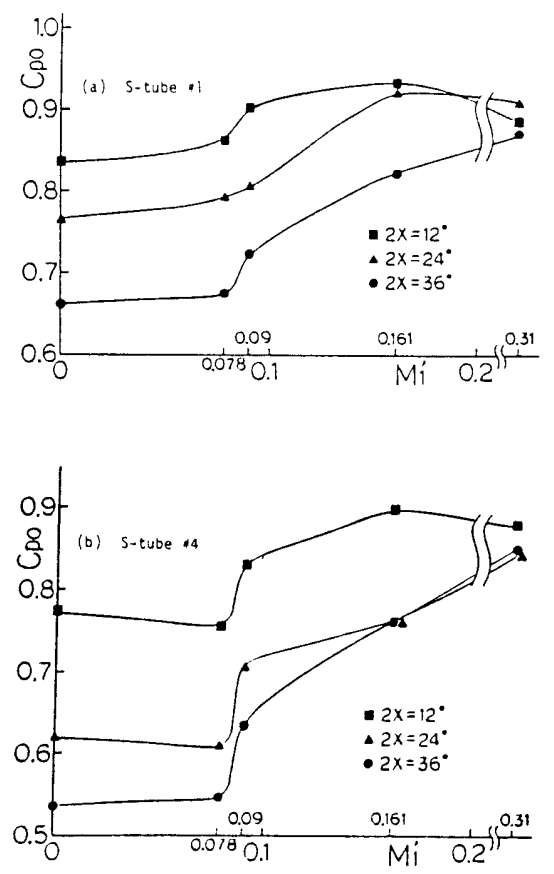

Fig. 6 Relation between one-dimensional pressure recovery performance $C_{p o}$ and swirl intensity of inlet flow $\mathrm{M}_{1}^{\prime}$ at the inlet section (with 3 kinds of inlet conical diffuser)
0.94 at $M_{1}^{\prime}=0.16$. In the case of $\# 4 \mathrm{~S}$-draft tube with $2 \chi=12^{\circ}, C_{p o}$ is 0.78 at $M_{1}^{\prime}=0$, and $C_{p o}$ reaches 0.9 at $M_{1}{ }^{\prime}=0.16$. Even at $M_{1}^{\prime}=0, C_{p o}$ is significantly improved when compared with $C_{p o}$ at $2 \chi=24^{\circ}$. In the case of $2 \chi=36^{\circ}$, both draft tubes show decreased $C_{p o}$ values. Thus, the pressure recovery performance of a draft tube, similarly to a draft tube with normal curvature, remarkably depends on the divergent angle of the conical straight portion at the tube inlet. Our study has revealed that the pressure recovery performance, degraded due to a sharp curvature, can be improved effectively by adjusting the angle.

\section{3 Relationships among length of inlet straight conical diffuser, friction loss of wall and loss of separation}

The relationship between losses of separation and wall friction will be discussed. As described above, initially, the performance of pressure recovery is largely affected by the scale of separation. However, the performance of pressure recovery is controlled by wall friction. As an example, $C_{p o}$ of $\# 1 \mathrm{~S}$-draft tube with conical angle $2 \chi=12^{\circ}$ ( sign) at $M_{1}{ }^{\prime}=0.31$ in Fig. 6(a) is slightly smaller than that of the same diffuser with $2 \chi=24^{\circ}$.

The reason for this relationship is explained as follows.

The length of a straight conical diffuser with $2 \chi=$ $12^{\circ}$ is twice as long as that with $2 \chi=24^{\circ}$ because the outlet diameters of both conical diffusers are the same. As a result, the wall areas of both diffusers are different and wall friction loss of the conical diffuser with $2 \chi=12^{\circ}$ is larger than that with $2 \chi=24^{\circ}$.

Separation occurs at the inner corner of the first bend until swirl strength reaches a moderate value, and separation loss is larger than wall friction loss. However, separation at the corner will dissipate if swirl strength becomes too strong, eg., at $M_{1}^{\prime}=0.31$, and the separation loss is little. On the other hand, wall friction loss becomes large if swirl strength becomes strong; then, the main loss will be due to wall friction. However, the result of the \#4 S-draft tube with sharply bent corner is different from that of the \#1 S-draft tube with gently bent corner. In the - case of the \#4 S-draft tube, separation is not dissipated even when inlet swirl is strong, and separation - - loss becomes larger than that due to wall friction.

- In the case of the largest conical angle, $2 \chi=36^{\circ}$, separation usually occurs at the first bend corner. In both \# 1 and \#4 S-draft tubes, separation loss is the major loss.

5. 4 Relationship among wall pressure difference distribution, divergent angle, and swirl flow intensity at inlet

Pressure difference $\tilde{C}_{p}$ between the inlet No. 0 
pressure tap and No.n pressure tap on the upper or lower wall surface of the draft tube is now discussed (see Fig. 1 for pressure tap locations). The distributions of $\tilde{C}_{p}$ on both upper and lower wall surfaces of the $\# 4 \mathrm{~S}$-draft tube are shown in Figs. 7 and 8 . Figure 7 shows the pressure difference distribution on the upper wall surface.

The upper wall surface has a boundary layer, which is thinned due to the secondary flow produced at the first bend. Separation is rarely caused on the upper wall surface because the flow is pressed against it at the first bend. Therefore, the pressure distribution on the wall surface is directly influenced by expansion of the passage area and curvature of the wall surface.

Consequently, $\widetilde{C}_{p}$ increases at the central part of the first bend (outer side of lst bend), and decreases at the central part of the second bend (inner side of 2 nd bend). It increases gradually as the area increases where there is no curvature. This trend is observed for all $2 \chi=12^{\circ}, 24^{\circ}$ and $36^{\circ}$. The change of wall pressure from the maximum to the minimum at $M_{1}^{\prime}=0$ increases as the angle of $2 \chi$ increases. The intensity of swirl flow affects the upper wall pressure distribution more at $2 \chi=24^{\circ}$ and $2 \chi=36^{\circ}$ than at $2 \chi=12^{\circ}$. This is because separation in the case of $2 \chi=12^{\circ}$ is originally small at $M_{1}^{\prime}=0$, and therefore the separation-suppressing effect does not vary much, even if $M_{1}^{\prime}$ is increased. Contrary to this, because separation in the case of $2 \chi=24^{\circ}$ or $36^{\circ}$ is large at $M_{\mathrm{r}}^{\prime}=0$, the separation-suppressing effect at the first bend outlet becomes significant as the intensity of swirl flow increases: separation decreases and $C_{p o}$ increases when the swirl flow is intensive, and $C_{p o}$ decreases when the swirl flow is weak. Figure 8 shows the wall pressure difference distribution on the lower side. The

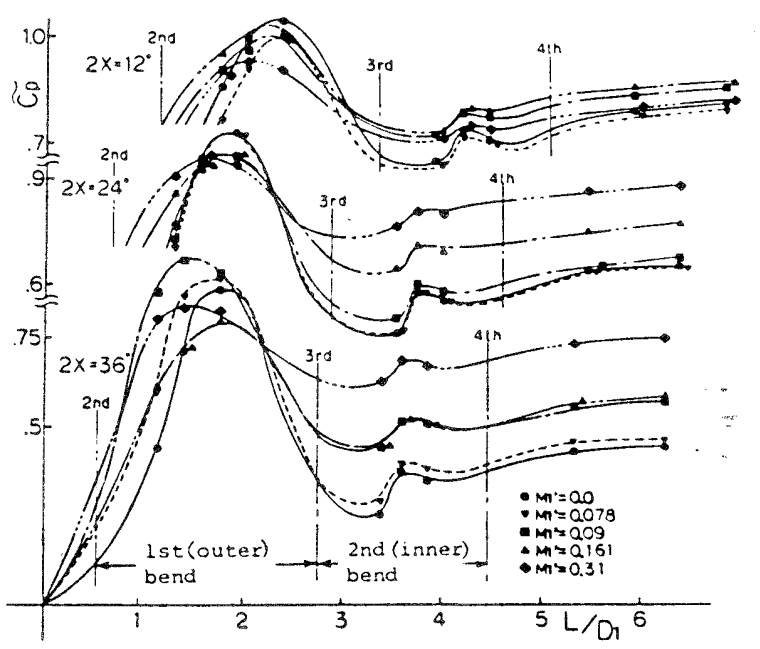

Fig. 7 Wall pressure distributions along upper contour of S-tube \#4 lower side wall contains the inner side of the first bend curvature, where large separation occurs. Due to this separation, the inner wall of the first bend curvature does not show a local pressure drop, which should be caused by the irregularity of wall, and $\tilde{C}_{p}$ increases downstream, comparatively sharply between the second and third sections, and gradually thereafter. In case the divergent angle of the inlet conical straight portion is as small as $2 \chi=12^{\circ}$, the swirl intensity affects the pressure recovery coefficient $\left(C_{p o}\right)$ insignificantly, but affects $C_{p o}$ increasingly as the angle increases to $2 \chi=24^{\circ}$ and $2 \chi=36^{\circ}$. In the case of $2 \chi=12^{\circ}$, pressure recovery becomes maximum at a medium swirl intensity of $M_{1}^{\prime}=0.16$. In case the angle increases to $2 \chi=24^{\circ}$ or $2 \chi=36^{\circ}$, the pressure recovery is maximum at the largest swirl intensity of $M_{1}^{\prime}=0.31$, similarly to Fig. 7. This phenomenon can not be explained easily: the separation produced on the inner side of the first bend curvature and the secondary flow are considered to be related in a complicated manner.

Furthermore, it is interesting that, although the reverse pressure gradient at the central part of the first bend curvature in Fig. 7 is larger than that in Fig. 8 , no separation is observed on the outer side of the first bend curvature; separation is observed only on the inner side of the first bend curvature. This can be explained as follows. Due to the secondary flow produced at the first bend, low-velocity fluid in the wall surface boundary layer is accumulated on the inner side of the curvature. The reverse pressure gradient acts on the mass of the accumulated lowvelocity fluid, causing the separation. Figure 9 shows photographs tracing the separation flow at $M_{1}^{\prime}=0.0$ on the wall surface. The separation zone is where alumi-

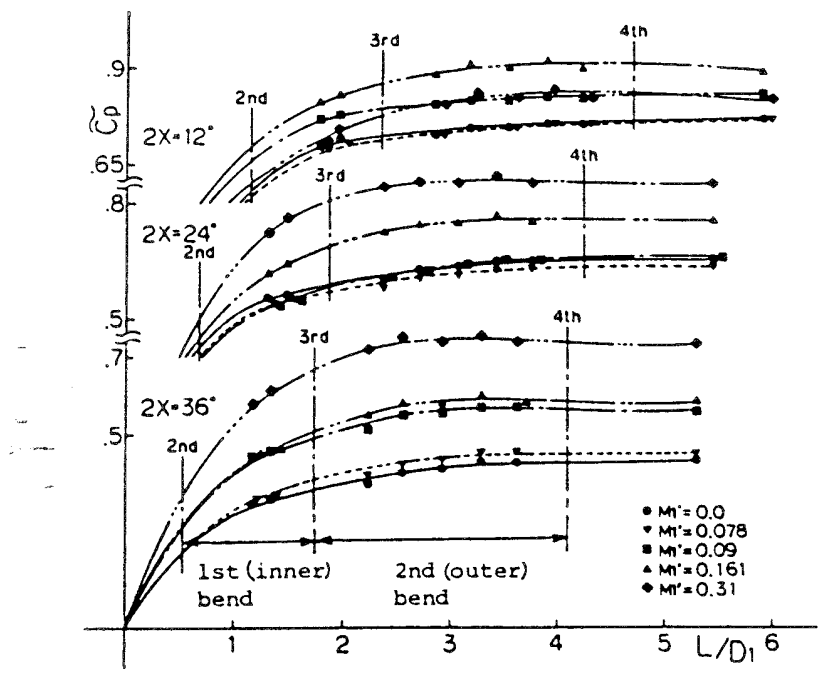

Fig. 8 Wall pressure distributions along lower contour of S-tube $\# 4$ 
num powder is accumulated at the bottom part of the wall. If three pictures of $2 \chi=12^{\circ}, 24^{\circ}$ and $36^{\circ}$ are compared, the largest separation zone is seen at $2 \chi=$ $36^{\circ}$. In this case, separation begins at the conical straight portion.

\subsection{Unsteady secondary flow in sharply bent S- draft tube}

In addition to the relationship between the pressure recovery coefficient curve and $M_{1}^{\prime}$, which is clearly observed in the visualized photographs, another interesting phenomenon was revealed during the experiment. In Fig. 4, we pointed out that $C_{p o}$ was sharply improved when $M_{1}^{\prime}$ increased slightly from 0.078 to 0.09 . The improvement of $C_{p o}$ resulted from a quick decrease of the separation zone area as mentioned previously. Immediately after the decrease in the separation area, a characteristically unsteady secondary flow, which is described below was observed.

Figure 10 presents visualized time-sequence photographs of the flow in the third section of the $\# 4 \mathrm{~S}$ draft tube at $M_{1}{ }^{\prime}=0.09$. The pictures were taken from down-stream side. These photographs, taken sequentially with an auto-drive camera, show the movement of tufts on the inner wall surface at the first bend. The pictures were taken at 0.4 -second intervals, or 10 frames for 3.6 seconds. The direction of swirl flow at the draft tube inlet is anti-clockwise looking upstream. Observing the movement of the tufts, in Pic. $1(t=0 \mathrm{~s})$, the direction of tufts on the left and right
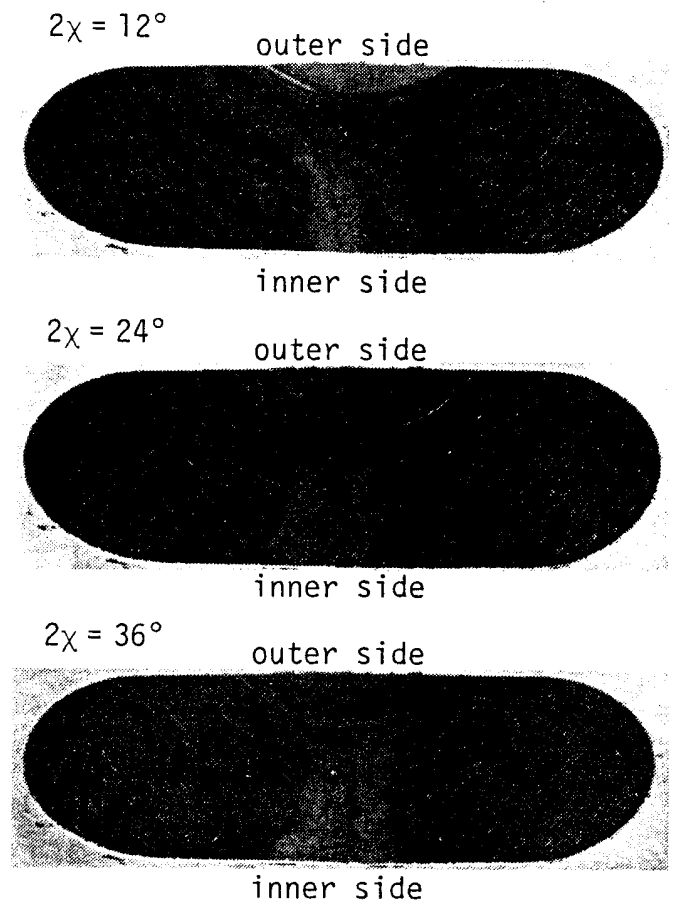

Fig. 9 Flow visualized results at 1 st bend (with 3 kinds of inlet conical diffuser), $M_{\mathrm{r}}^{\prime}=0$ $+$ side walls is downward and denotes the existence of a pair of symmetric secondary swirling flows, though the left side flow is more induced by the inlet swirl flow. Upon careful observation of Pic. $2(t=0.4 \mathrm{~s})$ and Pic. $3(t=0.8 \mathrm{~s})$, it is noted that the left side flow becomes less induced, the secondary flow with low energy develops along the left side wall and both wall sides with low- energy flow are accumulated along the center of the inner wall of the first bend. When the intensity of secondary flow along the left wall is balanced with that along the right wall, as shown in Pic. $4(t=1.2 \mathrm{~s})$, a large separation occurs at the center of the inner wall of the first bend. In Pic. 5 the left side secondary flow almost vanishes and at the next instant, in Pic. $6(t=2.0 \mathrm{~s})$, the induced flow appears on left side wall. The induced left side swirl flow sweeps off the low energy flow accumulated along the center of the inner wall and supplies the right side secondary flow as shown in Pic. $7(t=2.4 \mathrm{~s})$. In Pic. 8 , $(t=7.8 \mathrm{~s})$ the right side secondary flow recovers. In Pic. $9(t=3.2 \mathrm{~s})$ and Pic. $10(t=3.6 \mathrm{~s})$ the left side secondary flow prevails. The flow pattern in Pic. 4 is similar to that in Pic. 1 in Fig. 4. Picture 10 corresponds to a strong swirl flow. As observed above, a pair of secondary flows in the first bend of the S-draft tube fluctuate intermittently, changing mainly the intensity of the secondary flow having the same direction as that of the inlet swirl flow. A more interesting fact is that although the direction of swirl flow at the draft tube inlet is anti-clockwise looking upstream, clockwise secondary flow develops rather consistently. From the pressure recovery coefficient in Fig. 4 and the series of pictures in Fig. 10, the flow flucutuating intermittently at $M_{1}^{\prime}=0.09$ can be understood as a transitional phenomenon between no swirl and strong swirl flow conditions.

Figure 11 shows power spectra of pulsating pres. sure on the No. 0 pressure hole shown in Fig. 1. Figure 11( a ) corresponds to an inlet swirl strength of $M_{1}{ }^{\prime}=$ 0.0 , and double spiral secondary flow occurs in the draft tube. Main frequency of pulsating pressure exists about $2 \sim 3 \mathrm{~Hz}$ and low. The changing cycle of swirl direction of unsteady secondary flow described in Fig. 10 is low frequency and about $2 \sim 3 \mathrm{~Hz}$.

On the other hand, Fig. 11 ( b ) shows the results - of strong inlet swirl, $M_{1}^{\prime}=0.31$. Separation does not occur at the first bend corner because strong onedirectional swirling flow occupies all of the section. As a result, pulsating pressure has a high frequency and is weak.

\section{Conclusions}

From the results mentioned above, the following conclusions have been reached. 

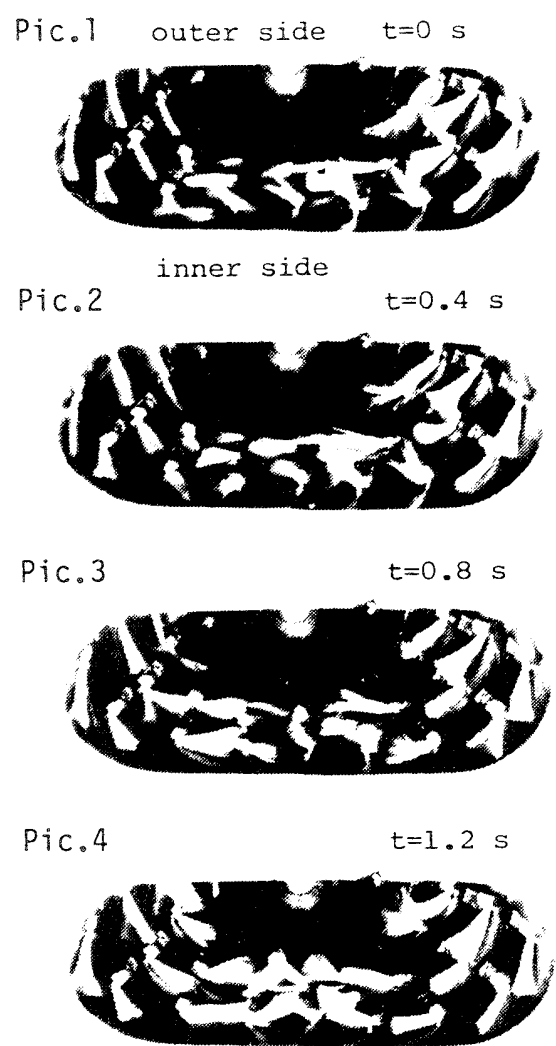

Pic. 5

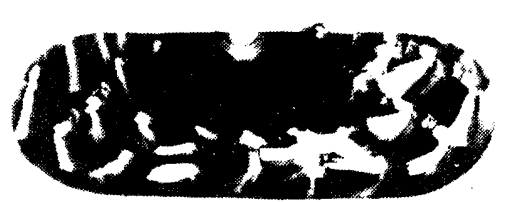

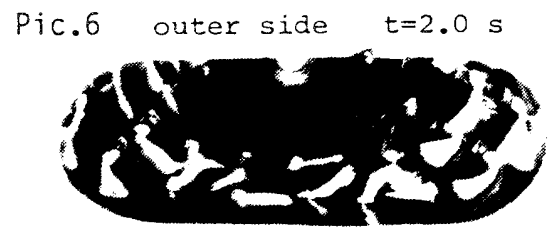

inner side

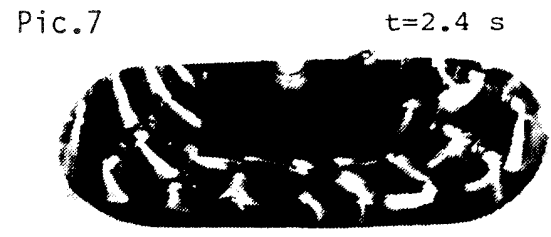

Pic. 8

$t=2.8 s$

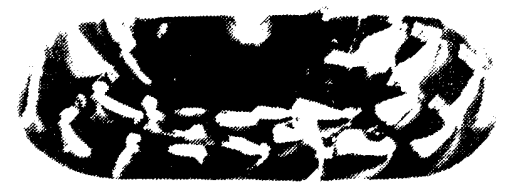

Pic.9

$t=3.2 \mathrm{~s}$

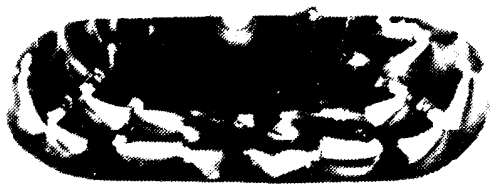

Pic. 10

$\mathrm{t}=3.6 \mathrm{~s}$

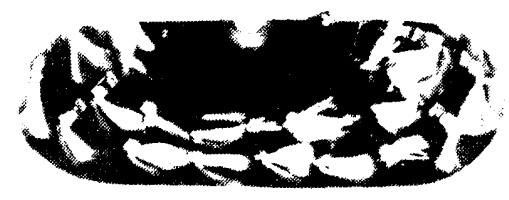

Fig. 10 A series of pictures showing behavior of the intermittent flow fluctuation observed in S-tube \# 4 with middle swirl intensity. The interval is $0.4 \mathrm{~s}$. Inner side of 1 st bend ( 3 rd section in Fig.1). $M_{\mathrm{i}}^{\prime}$ $=0.09$. Pictures were taken from down-stream side

(a) $M i=0.0$

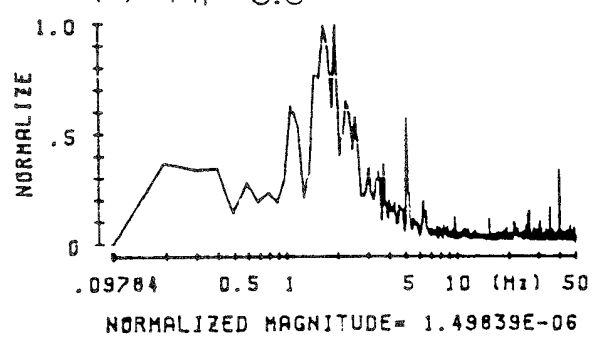

(b) $M i=0.31$

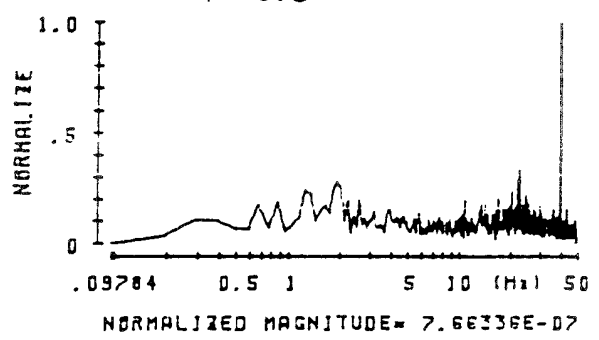

Fig. 11 Power spectrum distribution of pulsating pressure at No.0 section, in case of $\div 4$ S-draft tube, $2 \chi$ $=24^{\circ}$ 
(1) In the case where the upstream side bend of an S-draft tube is bent sharply, separation is produced at the inner side of the curvature, thus decreasing the pressure recovery performance.

(2) For improving the decreased pressure recov. ery performance, the following two methods were examined: (a) Increasing the swirl flow intensity at the draft tube inlet portion to some extent $\left(M_{1}^{\prime} \fallingdotseq 0.1\right)$; (b) Decreasing the divergent angle of the conical straight portion at the draft tube inlet to $2 \chi=12^{\circ}$. In this case, comparatively increased performance is observed even at $M_{1}^{\prime}=0$. Therefore, this method is practically useful.

(3) The performance of a sharply bent draft tube is low at $M_{1}^{\prime}=0 \sim 0.078$. It is improved sharply when the swirl flow intensity increases to a certain value
$\left(M_{1}^{\prime}=0.09\right)$ or higher. The flow pattern at the first bend immediately after the improvement of the performance is such that one of the pair of secondary flows which is in the same direction as the main swirl flow, appears, grows up, and disappears intermittently or repeatedly.

(4) Pulsating pressure shown in \# 4 S-draft tube was strong and of a low frequency if inlet swirl strength is zero or weak; $M_{1}^{\prime}<0.1$, and it has weak and high frequency if one is middle or strong.

\section{References}

(1) Shimizu, Y., Nakamura, F., Kubota, T. and Nakamura, S., Hydraulic Performance of S-Draft Tube for Low Head Axial Flow Turbine, The 12 th IAHR Symp., Stirling, (1984). 